After the expanded respiratory isolation policy was implemented, the number of tuberculosis episodes decreased from 4.4 per month (35 episodes among 103 patient admissions for TB over 8 months) to 0.6 per month (18 episodes among 358 patient admissions for smear-positive pulmonary TB over 28 months). There was an eightfold reduction in TST conversion rates among HCWs over the 2.5-year period. Six-month TST conversion rates decreased steadily from 3.3\% (118 conversions in 3,579 HCWs tested between January 1992 and June 1992), 1.7\%, $1.4 \%$, and $0.6 \%$ to just $0.4 \%$ (23 TST conversions among $5,153 \mathrm{HCW}$ s between January 1994 to June 1994; $P<.001)$.

The authors concluded that administrative controls appear to be the most important component of a tuberculosis program and should be the first focus of such a program, especially at public hospitals such as Grady Memorial where resources are most likely to be limited.

FROM: Blumberg HM, Watkins DL, Berschling JD, et al. Preventing the nosocomial transmission of tuberculosis. Ann Intern Med 1995;122:658-663.

\section{Elimination of Routine Ventilator Circuit Changes}

Dr. Marin Kollef and colleagues from Washington University School of Medicine, Barnes Hospital and Jewish Hospital, St. Louis, Missouri, recently conducted a study to determine whether the practice of not routinely changing ventilator circuits in patients who require prolonged mechanical ventilation was associated with an increased risk of nosocomial pneumonia.

In a randomized controlled trial in the intensive care unit (ICU) of two university-affiliated teaching hospitals, 300 patients admitted to an ICU and who required mechanical ventilation for more than 5 days were assigned randomly to receive either no routine ventilator circuit change or circuit change every 7 days. Ventilator-associated pneumonia rates were similar in both groups: 36 of 147 (24.5\%) patients receiving no routine changes developed pneumonia compared to 44 of the $153(28.8 \%)$ receiving changes every 7 days. In addition, there were no significant differences found between the treatment groups for hospital mortality, ICU mortality, death during mechanical ventilation, death in patients with ventilator-associated pneumonia, or mortality directly attributed to ventilator-associated pneumonia. Patients receiving circuit changes every 7 days had 247 circuit changes at a total cost of $\$ 7,410$, compared to $\$ 330$ in the patient receiving no routine changes.

The authors concluded that elimination of routine ventilator circuit changes can reduce medical care costs without increasing the incidence of nosocomial pneumonia. This study has significant implications for the hospital epidemiologist. Currently, because of the limitations in study design and the small number of patients prospectively examined in previous studies, the CDC, in their nosocomial pneumonia guidelines, could not give clear recommendation for the maximum length of time that ventilator circuits can be left in place safely during prolonged mechanical ventilation.

FROM: Kollef MH, Shapiro SD, Fraser VJ, et al. Mechanical ventilation with or without 7-day circuit changes. Ann Intern Med 1995;123:168-174.

\section{Albuterol-Associated Burkholderia cepacia}

An outbreak of Burkholderia (formerly Pseudomonas) cepacia respiratory tract colonization and infection in mechanically ventilated patients recently was reported by Dr. Richard Hamill and colleagues at the Veterans Affairs Medical Center and Texas Southern University in Houston. Forty-two patients had $B$ cepacia respiratory tract colonization or infection. Observation of intensive-care unit and respiratory-care personnel showed faulty infection control and respiratory therapy practices, including the following: (1) Respiratory therapists commonly carried a $10 \mathrm{~mL}$ bottle of albuterol in their pockets-frequently for several days at a time-and used it for multiple mechanically ventilated patients; (2) handwashing was not performed regularly; (3) when patients were being weaned from ventilators, the breathing circuits, including the in-line nebulizer, remained attached to the ventilator located at the bedside, and frequently these stand-by circuits were observed to be moist from condensation; and (4) the in-line nebulizers were not removed routinely from the circuit, rinsed or dried between treatment. In addition, at each successive treatment, the respiratory therapists added medication and diluent to the nebulizer reservoir without discarding the residual contents.

A case control study revealed that more case patients than controls received both nebulized albuterol and more respiratory therapy treatments. In-use albuterol solutions had $\mathrm{pH}$ values that were unstable, and benzalkonium chloride concentrations declined over time to levels capable of supporting bacterial growth. B cepacia was recovered from four of eight in-line medication nebulizer reservoirs or ventilation tubing and from two of two previously opened bottles of albuterol. Molecular fingerprints of patient isolates and environmental $B$ cepacia isolates were identical using repetitive-element polymerase chain reaction.

The authors point out that this outbreak emphasizes the importance of adherence to appropriate respiratory care and infection control practices, particularly when using multiple dose vials. Further, benzalkonium chloride may not be an appropriate medical preservative, because the agent did not provide effective bacteriostasis on numerous occasions.

FROM: Hamill RJ, Houston ED, Georghiou PR, et al. An outbreak of Burkholderia cepacia respiratory tract colonization and infection associated with nebulized albuterol therapy. Ann Intern Med 1995;122:762-766.

Additional news items in this issue: Microbe NamesAre You Keeping Up?, page 711; MRSA Outbreak Within a Family, page 724; HIV Transmitted by Bite, page 736. 